\title{
Benefits of Early in Postoperative Cardiac Surgery Patients
}

\author{
LE Angie Michelle May Gio루, LE Nallely Lugelia Tzel Kantún ${ }^{1}$, MCE Eloisa Beatriz del Socorro \\ Puch $\mathrm{Ku}^{* 2}$ and Laura Dioné Ortíz Gómez ${ }^{2}$ \\ ${ }^{1}$ Students of the Students of the Specialty in Intensive Therapy, Faculty of Nursing, Autonomous University of Yucatan, Mexico \\ ${ }^{2}$ Professor Faculty of Nursing, Faculty of Nursing, Autonomous University of Yucatan, Mexico
}

*Corresponding author: MCE Eloisa Beatriz del Socorro Puch Ku, Professor Faculty of Nursing, Faculty of Nursing, Autonomous

University of Yucatan, Mexico

\section{ARTICLE INFO}

Received: May 29, 2021

Published: 㓞 June 07, 2021

Citation: LE Angie Michelle May Gio, LE Nallely Lugelia Tzel Kantún, MCE Eloisa Beatriz del Socorro Puch Ku, Laura Dioné Ortíz Gómez. Benefits of Early in Postoperative Cardiac Surgery Patients. Biomed J Sci \& Tech Res 36(2)-2021. BJSTR. MS.ID.005833.

Keywords: Adult; Intensive Care Units; Heart/Surgery; Cardiac Surgical Procedures; Early Mobilization; Delayed Mobilization; Benefits

\section{ABSTRACT}

Introduction: After the review of the articles it was found that early mobilization in post operated patients, shorter duration in hospitalization and late complications after surgery.

Objective: Evaluate evidences of the benefits of early mobilization in post-cardiac surgery patients.

Methodology: The PRISMA (Preferred Reporting Items for Systematic Reviews and Meta-Analyses) methodology is followed, searches were done during the period from October 2020 to March 2021 in PubMed, portal of the Virtual Health Library, Science Direct. Cases and controls, case series, random clinical trials, systematic review were included, data were examined and extracted evaluating the quality with FLC 3.0 and the level and degree of evidence with the Sackett scale.

Conclusion: Early mobilization is safe under close monitoring of hemodynamics, improves functional capacity, decreased hospital stay and associated complications, preventing neuromuscular and cognitive disorders.

Abbreviations: CVD: Cardiovascular Diseases; EM: Early Mobilization; MV: Mechanical Ventilation; ECT: Extracorporeal Circulation Time; VHL: Virtual Health Library; BP: Blood Pressure, RPE: Rate of Perceived Exertion; ERAS: Enhance Recovery After Surgery

\section{Introduction}

Cardiovascular diseases (CVD) are one of the main public health problems worldwide. They are the main causes of preventable disease, disability and mortality. Worldwide, ischemic heart disease and cerebrovascular diseases accounted for just over $85 \%$ of all CVDs in 2015, which is equivalent to 15.2 million deaths [1]. In Mexico, CVDs are the main group of causes of death since 1990, the highest mortality has been observed in men. It has been seen that more than $60 \%$ of the adult population in the country has at least some CVD risk factor, which together with the aging process of the population generates, by itself, an increase in age-related diseases. This provides a future outlook in which mortality from these causes will tend to increase in the coming years [1]. Cardiac surgery has been the most successful treatment, due to advances in techniques and materials, which has resulted in safer procedures. Despite these advances, postoperative complications are frequent, and determinant for hospital stay and the limitation of functional recovery. In a study with 204 patients undergoing cardiac surgery, $58 \%$ had some postoperative complication, including pulmonary (31\%), cardiac (15.8\%) and neurological (13.9\%). Ribeiro et al, found a global mortality rate of $8 \%$, having pulmonary complications as one of its main causes. Early mobilization and respiratory physiotherapy are key factors for its prevention [2]. 
Bed rest has a negative effect on regional ventilation and the musculoskeletal system, with alterations in the architecture of the peripheral muscles and aerobic capacity, in addition to increasing insulin resistance. Bed rest is associated with imbalances in the production of pro and anti-inflammatory mediators and microvascular dysfunction. In the past, the mobility of patients undergoing cardiac surgery was restricted in order to reduce cardiac overload. Currently, multiple studies emphasize the importance of Early Mobilization (EM) [3] in the prevention of postoperative complications, improvement in functional capacity; reduction in hospital stay of patients, duration of delirium, use of Mechanical Ventilation (MV) and significant contribution to functional independence [2]. Despite this, there is no consensus regarding the onset time, types of mobilization, intensity, duration, hemodynamic and respiratory behavior and the impact of extracorporeal circulation time (ECT) on it. The definition of EM is complex and most of the studies do not mention it specifically: Achieving the bipedal with adequate orthostatic tolerance is gravitating to advance to new functional activities such as ambulation [2]. Articles have been described on the benefits of EM in adult patients after cardiac surgery. In a study published in 2017 by Ramos dos Santos, a systematic review analyzed nine articles, which revealed diversity in the techniques used for mobilization, as well as periods considered early for the start of the intervention. It was concluded that early mobilization seems to be important to prevent complications [3]. In 2019, Ramírez JI published a retrospective observational study in which it was concluded that EM in patients undergoing cardiac surgery is a safe and feasible intervention, since there are only significant variations in the first minute of the transitions and then behave without significant changes in the rest of the mobilization. It was interesting to observe that the higher TCEC (already previously described) was a limiting factor for the delay of EM [2]. Another study published in 2020 by Shirvani F, was a randomized, double-blind clinical trial, which concluded that early planned mobilization was effective in reducing delirium in postoperative patients undergoing coronary artery bypass graft [4]. The objective of this systematic review is to analyze the evidence for the benefits of early mobilization in patients after cardiac surgery.

\section{Methodology}

Systematic review with PRISMA methodology (Preferred Reporting Items for Systematic Reviews and Meta-Analyzes) [5]. The search stage was carried out during the period from October 5th, 2020 to March 14th, 2021; it consisted of the search for articles related to the topic: post-cardiac surgery patients hospitalized in the adult intensive care unit as well as early mobilization and late mobilization in hospitalized patients in the adult intensive care unit. The information on the aforementioned topic was investigated in different databases: Scielo, academic Google, PubMed, Lilacs, portal of the Virtual Health Library (VHL) Ciberindex Foundation, Redalyc, Science Direct, through the terms generated in the Descriptors in Health Sciences (DeCs) [6]. The aforementioned search was limited to research articles published in the Spanish, English, and Portuguese languages. We included in the search articles published since 2015 and that are post-cardiac surgery adult patients, articles from systematic reviews, cases and controls, cohorts, and clinical trials. Likewise, studies comparing early mobilization and late mobilization without being hospitalized in the adult intensive care unit, articles comparing early mobilization and late mobilization in patients who were not post-cardiac surgery operated, as well as articles that are low Quality was assessed using the methodological criteria established by the Critical Reading Skills Program of the Critical Reading Chips application (FLC 3.0). Likewise, studies comparing early mobilization and late mobilization without being hospitalized in the adult intensive care unit, articles comparing early mobilization and late mobilization in patients who were not post-cardiac surgery operated were excluded. Articles that are of low quality were also evaluated using the methodological criteria established by the Critical Reading Skills Program of the Critical Reading Fiches application (FLC 3.0) [7].

Similarly, those with a low level and degree of evidence were discarded using the Sackett scale [8], articles older than 5 years of publication and publications that speak of another population. To carry out the search, AND and NOT were used, as Boolean and/or positional operators, truncation operators $\left({ }^{*}\right)$ at the end of the root of the word and quotation marks, positional operators SAME, ADJ, WITH; for selection or discard of the articles was considered initially the reading of the titles and abstracts. In the bibliographic search, the analysis and preparation of the search was carried out with the following relevant terms: Adult Intensive Care Unit; Heart surgery; Early and late mobilization; Profits. To avoid bias, it was sought to incorporate the best possible quality in the search for studies, incorporating those that were most relevant to the subject of review, in the same way, complete studies were included, and those that did not represent the population were excluded. According to the search carried out in PubMed, BVS, and Science Direct, a critical reading was carried out to evaluate the quality of the evidence of the articles found, obtaining 50 articles of which 45 were excluded. The remaining five pieces of evidence: a randomized clinical trial, a cohort study, a case-control study, a systematic review, and a case series were accepted (Figure 1). 


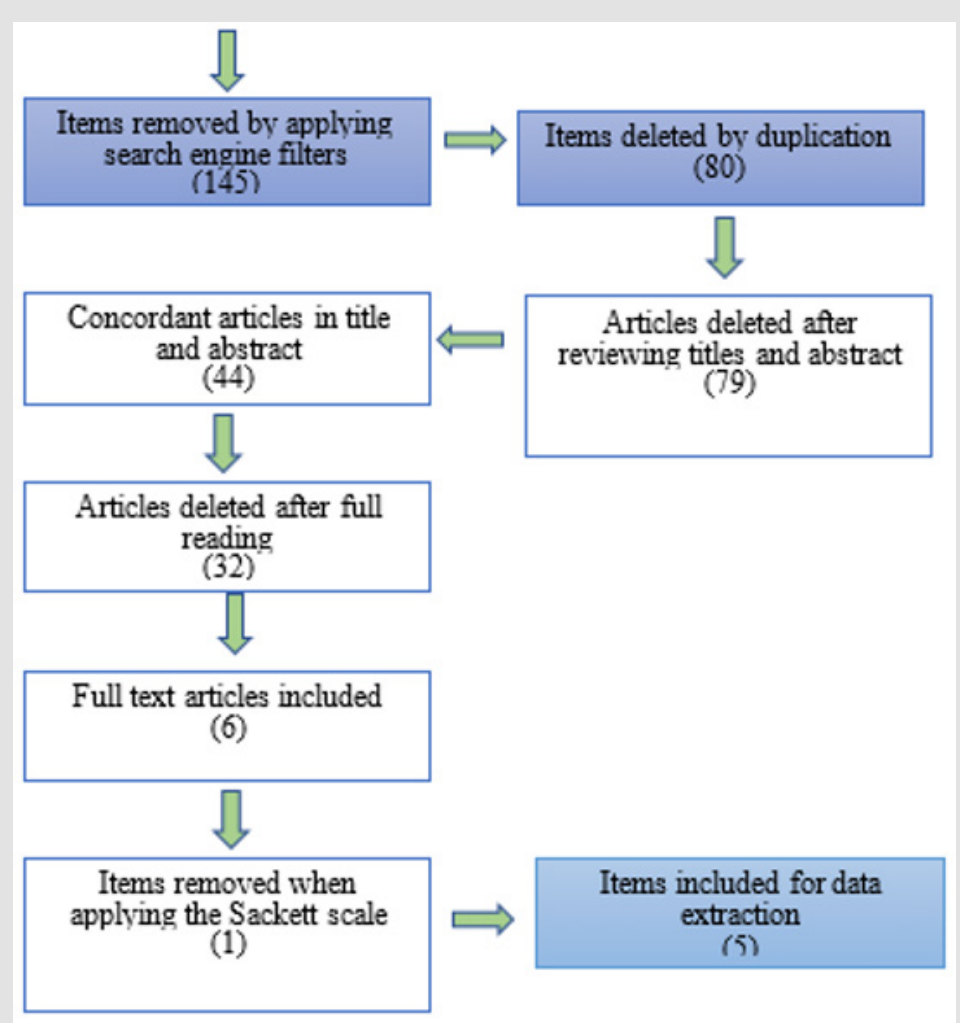

Figure 1: Summary of the screening of the selected articles.

\section{Development}

\section{Relevant Studies}

According to the search carried out in PubMed, BVS, and Science Direct, a critical reading was carried out to evaluate the quality of the evidence of the articles found, obtaining 50 articles of which 45 were excluded. The remaining five pieces of evidence: a randomized clinical trial, a cohort study, a case-control study, a systematic review, and a case series were accepted. The critical reading of the included studies was assessed using the methodological criteria established by the Critical Reading Skills Program of the application of FLC 3.0 [7]; it was conducted by two reviewers independently. After sharing the results, the disagreements that occurred were resolved through discussion, reaching consensus.

\section{Synthesis of the Evidence Found}

The analysis of the evidence of the articles found was organized according to the levels of evidence and degrees of recommendation according to the SACKETT scale [8]; as well as the database from which they were downloaded (Table 1). Next, the articles are broken down according to the objectives and the questions that can be answered.

Table 1: Titles of articles found, level of evidence and conclusions.

\begin{tabular}{|c|c|c|c|c|c|}
\hline Database & Paper Title & Type of Study & Evidence (scale) & Gradation & Conclusions \\
\hline Science Direct & $\begin{array}{c}\text { Effects of early } \\
\text { mobilization in } \\
\text { patients after cardiac } \\
\text { surgery: a systematic } \\
\text { review [3]. }\end{array}$ & Systematic review & Sackett & $\begin{array}{l}\text { Level of evidence: } 1^{\text {st }} \\
\text { Grade of } \\
\text { recommendation: A }\end{array}$ & $\begin{array}{l}\text { Regardless of the techniques } \\
\text { used as mobilization, the } \\
\text { fundamental thing is to avoid } \\
\text { bed rest. Early mobilization } \\
\text { appears to be important } \\
\text { to prevent postoperative } \\
\text { complications, improve } \\
\text { functional capacity, and reduce } \\
\text { the length of hospital stay in } \\
\text { patients after cardiac surgery. }\end{array}$ \\
\hline
\end{tabular}




\begin{tabular}{|c|c|c|c|c|c|}
\hline BVS & $\begin{array}{c}\text { Effects of the early } \\
\text { mobilization protocol } \\
\text { performed after } \\
\text { cardiac surgery on } \\
\text { patient care outcomes } \\
\text { [11]. }\end{array}$ & Cases and controls & Sackett & $\begin{array}{l}\text { Level of evidence: } 3 b \\
\text { Grade of } \\
\text { recommendation: }\end{array}$ & $\begin{array}{l}\text { Early mobilization is feasible in } \\
\text { adult cardiac surgery patients } \\
\text { and has significant benefits. } \\
\text { More research is recommended } \\
\text { on the effectiveness of early } \\
\text { mobilization in different groups } \\
\text { of patients. Health professionals } \\
\text { should be informed about } \\
\text { the importance of the early } \\
\text { mobilization protocol. Nurses } \\
\text { should be encouraged to } \\
\text { innovate to achieve successful } \\
\text { postoperative patient care } \\
\text { outcomes. }\end{array}$ \\
\hline PubMed & $\begin{array}{l}\text { Monitoring of oxygen } \\
\text { saturation and heart } \\
\text { rate during a single } \\
\text { early rehabilitation } \\
\text { session after cardiac } \\
\text { surgery [9]. }\end{array}$ & Case series & Sackett & $\begin{array}{l}\text { Evidence level: } 4 \\
\text { Grade of } \\
\text { recommendation: C }\end{array}$ & $\begin{array}{l}\text { Early rehabilitation after } \\
\text { cardiac surgery aims to prevent } \\
\text { immobilization, reduce the } \\
\text { effects of surgery on respiratory } \\
\text { function and facilitate the } \\
\text { recovery of autonomy in } \\
\text { activities of daily living (ADL) } \\
\text { after discharge. However, the } \\
\text { optimal perioperative physical } \\
\text { therapy care for patients } \\
\text { undergoing cardiac surgery is } \\
\text { not well established. Monitoring } \\
\text { peripheral oxygen saturation } \\
\text { and HR during and not just } \\
\text { before and at the end of a } \\
\text { standardized early rehabilitation } \\
\text { session helped us ensure the } \\
\text { safety of our protocol. }\end{array}$ \\
\hline PubMed & $\begin{array}{c}\text { Effects of the early } \\
\text { mobilization protocol } \\
\text { on cognitive outcome } \\
\text { after cardiac surgery } \\
{[10] .}\end{array}$ & $\begin{array}{l}\text { Randomized } \\
\text { controlled trial }\end{array}$ & Sackett & $\begin{array}{l}\text { Level of evidence: } 1 \mathrm{~b} \\
\text { Grade of } \\
\text { recommendation: A }\end{array}$ & $\begin{array}{l}\text { The implementation of the } \\
\text { early mobilization protocol has } \\
\text { positive effects on the cognitive } \\
\text { outcome and in the ICU after } \\
\text { cardiac surgery. }\end{array}$ \\
\hline PubMed & $\begin{array}{l}\text { Early mobilization } \\
\text { reduces delirium after } \\
\text { coronary artery bypass } \\
\text { graft surgery [4]. }\end{array}$ & $\begin{array}{l}\text { Randomized, double- } \\
\text { blind clinical trial }\end{array}$ & Sackett & $\begin{array}{l}\text { Level of evidence: } 1 \mathrm{~b} \\
\text { Grade of } \\
\text { recommendation: A }\end{array}$ & $\begin{array}{l}\text { Early planned mobilization } \\
\text { was effective in reducing } \\
\text { postoperative delirium in } \\
\text { patients undergoing coronary } \\
\text { artery bypass graft. }\end{array}$ \\
\hline
\end{tabular}

\section{Results}

Sala V [9] published a series of cases in which the population was 48 consecutive patients (35M), mean age 61 years, with cardiovascular disease (CVD), who underwent cardiac surgery. SpO2\%, HR, systemic blood pressure (BP), chest wound pain (VAS) and rate of perceived exertion (RPE) were monitored during the rehabilitation session, after weaning from oxygen therapy. In the results, during all phases the mean Sp02 was 94\% ( \pm 1.8$)$ and the mean HR was $85 \mathrm{bpm}( \pm 13.3)$. The number of desaturation events was 14 in total and the mean time with Sp02 $<90 \%$ was $3( \pm 6.5)$ during the entire rehabilitation session. In addition, the mean arterial pressure (MAP) after reaching the sitting position was $124.7( \pm 11.9) / 78.6( \pm 8.4)$ and after ambulation it was $131.5( \pm$ $11.5) / 82.9$ ( \pm 7.3 ). It was concluded that monitoring of peripheral oxygen saturation and HR (heart rate) during the rehabilitation session and not just before and at the end of a standardized early session helped to ensure the safety of the protocol [9]. Ziyaeifard, et al. [10] published that 80 patients were recruited. All patients, who were candidates for cardiac surgery from March to September 2016, were evaluated based on the inclusion criteria before and after surgery. For patients who consented to participate, the MMSE (Mini-Mental State Examination) questionnaires were completed. The mean score of all participants was reported as 28.74 at baseline (SD: 1.54; SE: 0.172) with no significant difference between the intervention and control groups $(\mathrm{P}=0.318)$. The patients had significantly higher scores on the second postoperative day and at the time of discharge from the ICU. In the intervention group (P $<0.001$ ) there was no significant relationship between preoperative cognitive impairment and allocation to intervention or control group ( $\mathrm{P}>0.05$ ). However, there was a significant relationship between cognitive impairment on the second postoperative day and at the time of discharge from the ICU, and the allocation group $(\mathrm{P}<0.05)[10]$. On the second postoperative day, time awareness, place awareness, attention and calculation, recent memory, and tongue function scores were significantly higher in the intervention group ( $\mathrm{P}<0.05)$. Registration was also significantly better in the intervention group at discharge from the ICU $(\mathrm{P}=0.041)[10]$. 
Duration of intubation and ICU stay were significantly longer in the control group ( $\mathrm{P}=0.017 ; \mathrm{P} \leq 0.001)$. 34 patients $(85 \%)$ in the intervention group did not experience any cognitive impairment and only 15\% (6 patients) had mild cognitive impairment at discharge. Moderate, mild and non-cognitive prevalence. Impairment was reported to be $5 \%$ (two patients), 50\% (20 patients), and $45 \%$ (18 patients), respectively; however, decreasing the intubation period and implementing progressive early mobilization could lead to a decrease in the stay in the ICU. In conclusion, progressive early mobilization can be part of an enhanced recovery after surgery (ERAS) (Enhance Recovery After Surgery) that are useful in a variety of surgical procedures and have benefits on patients, especially in their cognitive function [10]. Shirvani F [4] found that patients in the intervention group had normal function on the second postoperative day compared to the control group (25 vs. 2 , respectively, $\mathrm{p}=0.001$ ). The intervention group had significantly higher Neecham scores on postoperative day 2 (22.49 \pm 2.03 vs $26.82 \pm 2.10, \mathrm{p}=0.001)$. The multivariate analysis showed significant associations between the Neecham score and age $(p$ $=0.022)$, ejection fraction $(\mathrm{p}=0.015)$, myocardial infarction $(\mathrm{p}=$ $0.016)$, systolic pressure $(p=0.009)$ and diastolic pressure $(p=$ 0.008 ). It is concluded that early planned mobilization was effective in reducing postoperative delirium in patients undergoing coronary artery bypass graft [4].

\section{Days of Hospital Stay}

Ramos Dos Santos PM, et al. [3] mentions that they selected nine trials. The PEDro scale showed that the studies had a low risk of bias (range 5 to 9 points). The trials revealed diversity in the techniques used for mobilization, as well as periods considered early for the start of the intervention. The early mobilization groups had better results compared to the untreated control groups [3]. Yayla A, et al. [11] indicated that the participants were patients who underwent cardiac surgery between January and October 2015. The study included 102 patients (51 patients each in the experimental and control groups). The introductory characteristics form, the Richards-Campbell Sleep Questionnaire (RCSQ), length of hospital stay (postoperative), and the development of a late postoperative complications form were used to collect data. Study results revealed that patients in the experimental group had better improvement in RCSQ scores, shorter duration of hospitalization, and fewer late complications after surgery than patients in the control group [11]. Early mobilization is feasible in adult cardiac surgery patients and has significant benefits, including better improvement, shorter length of hospitalization, and fewer late complications after surgery, it contributes to improving perioperative functional status in elderly patients, reduce the heart rate, this being a vital aspect to reduce myocardial pressure. Finally, it should be mentioned that more research is recommended on the effectiveness of early mobilization in different groups of patients. The PRISMA methodology has been used to demonstrate procedures that today are part of daily nursing practice; As in the case of the present study, we can consider that the results are reliable and with a small margin of bias [5]. Every client, user or person in the AICU requires evidence-based care with the highest possible quality and safety, [12] including early mobilization, which was the central theme in this work.

\section{Review Contributions}

The results of this review showed that the mobilizations in bed of post-cardiac surgery patients, the mean Spo2 and the mean HR were not altered; however, when sitting and walking, the parameters were not significantly altered $[9,13]$. The length of stay in the AICU did not increase [3] cognitive status, nor did it decrease [10]. An included article also showed no increase in functional capacity and postoperative complications due to early mobilization [3]. In a systematic review prepared by Arias Fernández P [14] in 2018, the following benefits were reported for mobilizing hospitalized patients in the AICU, where a significant effect was obtained on functional status, muscle strength, duration of mechanical ventilation, the ability to walk to discharge and the quality of life in health. It should be noted that the patients in the aforementioned study were not defined as post-cardiac surgery patients and patients hospitalized in AICU were considered regardless of their main diagnosis [14]. One aspect that Aria Fernández [14] does not report and that is currently reported is the improvement in sleep quality, shorter hospital stay and fewer late complications after surgery [11]. The present review shows that early mobilization had a significant effect on functional status, muscle strength, duration of mechanical ventilation, ability to walk at discharge, and health quality of life in post-cardiac surgery patients $[3,9,10,11]$. Similar to what was found, Azevedo, Paulo; Gomes and Barbara [15] included six studies in their systematic review, showing that early mobilization can facilitate the functional rehabilitation of these patients, promoting gains in muscle strength and greater participation in activities of daily living [15].

Despite the benefits of early mobilization in post-surgery patients, there are studies such as that by Wang J, et al. [16] where there is evidence that nurses had a good knowledge (more than $96.5 \%$ ) of the benefits and indications for stopping early mobilization; Wang J, et al. [16] point out that the main barriers perceived by the nursing staff to carry out mobilization in postoperated cardiac surgery patients were: very heavy workload (76.8\%), insufficient equipment and devices (50.2\%), lack of written protocols or guidelines (50.2\%), inadequate training (47.1\%), potential occupational hazards (42.3\%) [16]. In short, adopting new measures based on evidence always brings changes in hospital environments that can go against daily routines, particularly in post-cardiac surgery patients [12]. 


\section{Study Limitations}

In the search for information, no evidence was found in the master catalog of clinical practice guidelines [17] regarding early mobilization in post-operated cardiac surgery patients or in patients hospitalized in the Adult Intensive Care Unit, so this work is relevant from the practical, administrative and care point of view.

\section{Conclusion}

To reach the conclusion, the publication of a systematic review was taken into account in two articles in which no differences in results were found between the control group and the intervention group with respect to extubation time, incidence of complications, capacity functional and length of hospital stay; [13] however, taking into account most of the articles consulted, it is concluded that early mobilization is feasible and safe in post-cardiac surgery patients who are in the intensive care unit under close monitoring of hemodynamics since it improves functional capacity, reduces hospital stay and associated complications, thus preventing neuromuscular disorders, does not significantly alter the patient's hemodynamics, helping to prevent cognitive decline.

\section{Authorship Contribution}

Angie Michelle May Gio first author; Nallely Lugelia Tzel Kantún, second author, Eloisa Beatriz del Socorro Puch Ku y Laura Dioné Ortíz Gómez, acted as adviser to the systematic review, four author.

\section{References}

1. Davila c (2019) Tendencia e impacto de la mortalidad por enfermedades cardiovasculares en México, 1990-2015. Rev Cuba Salud Pública 45(4): 1081.

2. Ramírez JI, Grimalt R, Espinoza C, Jalil Y, Ramírez JI, et al. (2019) Comportamiento hemodinámico y respiratorio durante la movilización temprana de pacientes sometidos a cirugía cardíaca: Experiencia en un Hospital Público. Rev Chil Cardiol 38(3): 190-197.

3. Ramos dos Santos PM, Aquaroni Ricci N, Suster E, Paisani D, Dias Chiavegato L (2017) Effects of early mobilisation in patients after cardiac surgery: a systematic review. Physiotherapy 103(1):1-12.

\section{ISSN: 2574-1241}

DOI: $10.26717 /$ BJSTR.2021.36.005833

MCE Eloisa Beatriz del Socorro Puch Ku. Biomed J Sci \& Tech Res

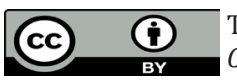

This work is licensed under Creative Commons Attribution 4.0 License

Submission Link: https://biomedres.us/submit-manuscript.php
4. Shirvani F, Naji SA, Davari E, Sedighi M (2020) Early mobilization reduces delirium after coronary artery bypass graft surgery. Asian Cardiovasc Thorac Ann 28(9): 566-571.

5. Moher D, Shamseer L, Clarke M, Ghersi D, Liberati A, et al. (2016) Ítems de referencia para publicar Protocolos de Revisiones Sistemáticas y Metaanálisis: declaración PRISMA-P 2015 . Revista Española de Nutrición Humana y Dietética . scieloes 20(2): 148-160.

6. DeCS - Descriptores en Ciencias de la Salud.

7. López M, Reviriego E, A G, J B (2020) Actualización del Sistema de Trabajo Compartido para Revisiones Sistemáticas de la Evidencia Científica y Lectura Crítica (Plataforma FLC 3.0).

8. Manterola C, Asenjo Lobos C, Otzen T (2014) Jerarquización de la evidencia: Niveles de evidencia y grados de recomendación de uso actual. Rev chil infectol 31(6).

9. Sala v, Petrucci L, Monteleone s, Conte T, Carlisi E, et al. (2016) Oxygen saturation and heart rate monitoring during a single session of early rehabilitation after cardiac surgery 52(1): 12-19.

10. Ziyaeifard M, Gholami B, Fatemeh L, Azarfarin S, Reza Aminnejad, et al. (2018) Effects of Early Mobilization Protocol on Cognitive Outcome after Cardiac Surgery. Ann Anesthesiol Crit Care 3(1): e63594.

11. Ayşegül y, Nadiye Ö (2019) Effects of early mobilization protocol performed after cardiac surgery on patient care outcomes. Int J Nurs Pract 25(6): e12784.

12. Castro M, Simian D (2018) La Enfermería Y La Investigación. Rev Médica Clínica Las Condes 29(3): 301-310.

13. Alves da Cruz MM, Ricci Vitor AL, Bonini Borges GL, Fernanda da Silva P, Ribeiro F, et al. (2020) Acute Hemodynamic Effects of Virtual RealityBased Therapy in Patients of Cardiovascular Rehabilitation: A Cluster Randomized Crossover Trial. Arch Phys Med Rehabil 101(4): 642-649.

14. Arias Fernández P, Romero Martin M, Gómez Salgado J, Fernández García D (2018) Rehabilitation and early mobilization in the critical patient: systematic review. J Phys Ther Sci 30(9): 1193-1201.

15. Azevedo P, Gomes B (2015) Effects of early mobilisation in the functional rehabilitation of critically ill patients: a systematic review. Rev Enferm Ref (IV Série): 129-138.

16. Wang J, Xiao Q, Zhang C, Jia Y, Shi C (2020) Intensive care unit nurses' knowledge, attitudes, and perceived barriers regarding early mobilization of patients. Nurs Crit Care 25(6): 339-345.

17. Motor de búsqueda CMGPC - Catálogo Maestro.

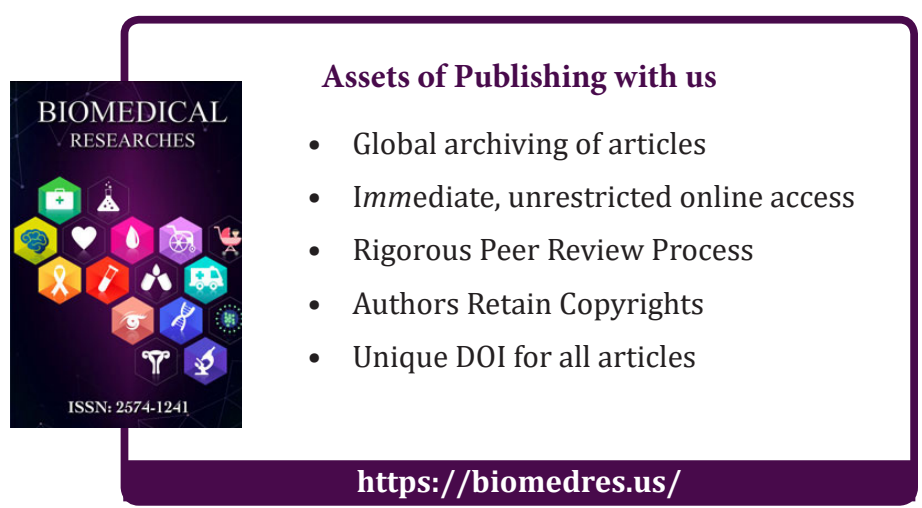

